\title{
Classificações de enfermagem: mapeamento entre termos do foco da prática
}

\author{
Nursing classification: cross-mapping between focus axis terms
}

Clasificaciones de enfermería: mapeamento entre los términos del foco de la práctica

\section{Romana Reis da Silva', Andreia Malucelli', Márcia Regina Cubas'}

'Pontifícia Universitária Católica do Paraná. Pós-Graduação em Tecnologia da Saúde. Curitiba, PR

Submissão: 29/08/2008

Aprovação: 22/1 1/2008

\section{RESUMO}

Devido às mudanças estruturais das classificações: CIPE ${ }^{\circledR}$ beta 2, versão 1.0 e CIPESC ${ }^{\circledR}$, este estudo descritivo tem como objetivo construir um mapeamento entre os termos do foco da prática, identificando semelhanças e diferenças. A CIPE ${ }^{\circledR} 1.0$ em relação à beta 2 apresenta: $41 \%$ dos termos novos; $33 \%$ idênticos; $4 \%$ com conceito ampliado; $12 \%$ modificados; $8 \%$ diferentes; e $2 \%$ conceituados apenas na beta 2. A CIPE ${ }^{\circledR} 1.0$ em relação à CIPESC ${ }^{\circledR}$ apresenta: 79\% são novos; 8\% idênticos; 1\% ampliado; 5\% modificados; 4\% diferentes; e $3 \%$ sem conceito. Houve dificuldades oriundas do processo de tradução e editoração da CIPE ${ }^{\circledR} 1.0$ e da inexistência de conceitos em alguns termos da $\mathrm{CIPESC}^{\circledR}$. Este trabalho desencadeará um processo de validação dos termos não equivalentes.

Descritores: Classificação; Enfermagem; Vocabulário controlado.

\section{ABSTRACT}

Due to structural changes of classifications: ICNP® beta-2, version 1.0 and CIPESC $®$, this descriptive study aims to identify similarities and differences by building a map between the terms of the focus of practice. The ICNP ${ }^{\circledR} 1.0$ on the beta- 2 shows: $41 \%$ are new terms, $33 \%$ identical, $4 \%$ with expanded concepts, $12 \%$ modified; $8 \%$ different and $2 \%$ there are concepts only in beta- 2 . The ICNP ${ }^{\circledR} 1.0$ in relation to CIPESC ${ }^{\circledast}$ presents: $79 \%$ are new; $8 \%$ identical; 1\% extended; $5 \%$ modified; $4 \%$ different and $3 \%$ without concept. There were difficulties from the process of Brazilian translating and publishing of ICNP ${ }^{\circledR} 1.0$ and the absence of some concepts in terms of CIPESC ${ }^{\circledast}$. This work will trigger the validation process for non equivalent terms.

Descriptors: Classification; Nursing; Vocabulary, controlled.

\section{RESUMEN}

Debido a los cambios estructurales de las clasificaciones: CIPE ${ }^{\circledR}$ beta-2, versión 1.0 y CIPESC $^{\circledast}$, este estudio descriptivo tiene como objetivo construir un mapeamiento entre los términos del foco de la práctica, identificando semblenzas y diferencias. La CIPE ${ }^{\circledR} 1.0$ en relación a beta- 2 presenta: $41 \%$ de los términos nuevos; $33 \%$ idénticos; $4 \%$ con el concepto ampliado; $12 \%$ modificados; $8 \%$ diferentes y $2 \%$ conceptuados solamente en la versión beta-2. La CIPE ${ }^{\circledR} 1.0$ en relación a CIPESC ${ }^{\circledR}$ presenta: $79 \%$ son nuevos; $8 \%$ idéntico, $1 \%$ ampliado; $5 \%$ modificados; $4 \%$ diferentes y $3 \%$ sin concepto. Hubieron dificultades provenientes del proceso brasileiro de traducción y editoración de la CIPE $^{\circledR} 1.0$ y de la ausencia de algunos conceptos en términos de CIPESC ${ }^{\circledR}$. Este trabajo va a iniciar un proceso de validación de los términos no equivalentes.

Descriptores: Clasificación; Enfermería; Vocabulario controlado. 


\section{INTRODUÇÃO}

A prática da enfermagem faz uso de uma vasta Quantidade de informações Que constam no prontuário do paciente: suas Queixas, dados clínicos, diagnósticos, intervenções e resultados de enfermagem. No entanto, essas informações não estão estruturadas ou documentadas, de modo a sistematizar o cuidado e demonstrar a importância da Enfermagem nos serviços de saúde e, conseQüentemente, sua contribuição para a Qualidade da assistência ao ser humano(l).

A sistematização do cuidado é um conjunto de ações Que se inter-relacionam visando à assistência de Enfermagem. Esta interação é denominada "processo de enfermagem", Que é um instrumento dinâmico Que auxilia na construção do plano assistencial e, para Wanda Horta ${ }^{(2)}$, é composto de 6 fases: histórico do paciente, diagnóstico de enfermagem, plano assistencial, prescrições de enfermagem, evolução e prognóstico.

A organização da assistência ao ser humano é de suma importância, pois influencia diretamente na Qualidade de atendimento, na segurança dos cuidados prestados, na descrição da prática de enfermagem, na comunicação entre os enfermeiros e entre estes e outros profissionais ${ }^{(3)}$.

Uma das dificuldades da sistematização do cuidado e da visibilidade da profissão é Que a linguagem usada para descrever as atividades realizadas pelo enfermeiro muda no decorrer dos tempos e nos diferentes contextos culturais, resultando em diferentes compreensões de um mesmo fenômeno(4).

Os esforços para mudar esta realidade culminaram a partir da década de 1950, em escala mundial, com intuito de desenvolver modelos conceituais e teorias, a fim de identificar conceitos específicos da profissão e seu emprego na prática; porém, eram movimentos isolados. A partir da década de 1970, os movimentos começaram a unificar-se e, nos anos de 1990, este assunto ganhou força $a^{(5)}$.

Atualmente, existem várias classificações, cada uma se diferenciando em determinadas fases do processo de enfermagem. Este estudo deter-se-á nas classificações CIPE ${ }^{\circledR}$ (Classificação Internacional para a Prática de Enfermagem) e CIPESC ${ }^{\circledR}$ (Classificação Internacional para a Prática de Enfermagem em Saúde Coletiva), Que caracterizam o diagnóstico, intervenção e resultado de enfermagem, os Quais, em conjunto, determinam o plano de cuidados, direcionando assim a assistência ${ }^{(6)}$.

Esta pesquisa é parte integrante do projeto de pesquisa denominado "Compondo uma Nova Geração de Sistemas Classificatórios para as Práticas de Enfermagem", do Programa de Pós-graduação em Tecnologia em Saúde da Pontifícia Universidade Católica do Paraná, Que tem como objetivo geral avaliar a aplicabilidade de um sistema computacional para auxílio ao raciocínio diagnóstico individual e coletivo com uso da $\mathrm{CIPE}^{\circledR} / \mathrm{CIPESC}^{\circledR}$.

A CIPE ${ }^{\circledR}$ representa uma ferramenta Que produz informações para a tomada de decisão do enfermeiro, através de uma linguagem de enfermagem unificada e universal. Trata-se de uma terminologia combinatória Que permite formular um diagnóstico de enfermagem, delinear intervenções e identificar resultados aos cuidados prescritos. Estas ações desencadeiam informações Que contribuirão na formulação de políticas de saúde, na contenção de custos, na informatização dos serviços de saúde, no controle do próprio trabalho de enfermagem e nos avanços da profissão $0^{(7)}$.
A CIPE ${ }^{\circledR}$ é um instrumento dinâmico e mutável e, para sua manutenção, é preciso uma constante avaliação, bem como revisão e validação dos termos, para reduzir a ambigüidade e redundância ${ }^{(8)}$. $\mathrm{O}$ fruto deste constante aperfeiçoamento resultou em Quatro versões: CIPE ${ }^{\circledR}$ versão alpha ${ }^{(9)}(1996)$, versão beta (1999), beta$2^{(10)}(2001)$ e versão 1.0 $0^{(11)}(2005)$. Esta última aponta para a construção de uma ontologia, a partir da inferência hieráreuica entre os termos. Entende-se por ontologia a maneira de representar o conhecimento de forma organizada, a fim de facilitar a compreensão, permitir o compartilhamento das informações e construir uma base de conhecimento ${ }^{(12)}$.

Faz-se necessário a atualização da $\operatorname{CIPESC}^{\circledR}$, bem como a compreensão e adeQuação da nova estrutura da CIPE ${ }^{\circledR}$, uma vez Que a CIPESC $^{\circledR(13)}$ teve como base para sua estruturação a CIPE ${ }^{\circledR}$ versão beta.

Neste contexto, o propósito deste estudo é construir um mapeamento entre os termos do foco da prática da CIPE ${ }^{\circledR}$ beta-2, da CIPE $^{\circledR} 1.0$ e da CIPESC $^{\circledR}$, identificando semelhanças e diferenças entre os mesmos.

\section{REVISÃO DA LITERATURA}

\section{CIPE $^{\circledast}$}

O surgimento da $\mathrm{CIPE}^{\circledR}$ ocorreu em resposta às necessidades reconhecidas da profissão, a partir de 1989. As associaçõesmembro do Conselho Internacional de Enfermeiras (CIE) desencadearam estudos para viabilizar o projeto de desenvolver um vocábulo de termos estruturados para descrever e organizar a prática de enfermagem em uma linguagem universal ${ }^{(14)}$.

O primeiro resultado deste projeto foi uma listagem de termos, em 1993, Que se baseou em um levantamento internacional dos sistemas de classificação de enfermagem já em uso. Em 1996, foi lançada a CIPE $^{\circledR}$ versão alpha, cuja estruturação foi realizada em três etapas: a identificação dos termos no âmbito internacional; a conceitualização destes termos e a estruturação dos termos, hierarQuizando-os em três pirâmides ou modelos. A pirâmide inicial, construída como uma classificação monoaxial em cujo ápice encontra-se um único princípio geral de divisão, descreve 292 fenômenos de enfermagem, Que representa o domínio do cliente (ser humano ou meio ambiente). A segunda pirâmide, construída como uma classificação multiaxial na Qual o termo subdivide-se em seis eixos, descreve as intervenções de enfermagem, Que representa o domínio das ações realizadas pelos enfermeiros. A terceira pirâmide não foi desenvolvida e visava descrever os resultados clínicos do trabalho da enfermagem ${ }^{(0,15)}$.

Com a utilização da versão alpha, surgiram outras necessidades enviadas ao CIE, determinantes para a edição da versão beta, lançada em 1999. Nesta versão, o enfoque da classificação dos fenômenos de enfermagem passa a ser multiaxial e constituída por oito eixos. Isto permite mais de uma divisão do termo superior e também combinações de conceitos de diversas divisões e eixos, tornando assim o instrumento mais flexível e com maior liberdade para realizar combinações entre os termos, por exemplo: os conceitos simples como "sono" e "perturbado", se combinados, formam um conceito complexo, isto é, "sono perturbado". Em outros sistemas classificatórios, esta combinação de conceitos não acontece, pois já são existentes como conceitos pré-combinados ${ }^{(16)}$. 
Em 200 I, foi lançada a versão beta-2, com alterações, correções e inclusão de alguns códigos, conceitos e termos. A mais nova versão da CIPE ${ }^{\circledR}$ - a versão 1.0, lançada em 2005, é resultado de mudanças de critérios para o estudo das classificações, almejando não apenas uma simples linguagem unificada, mas uma linguagem completa e sofisticada, apoiada na utilização de software de sistemas de informações, de modo a simplificar a representação da classificação e evitar redundância e ambigüidade entre os termos.

A CIPE ${ }^{\circledR} 1.0$ apresenta modificações na estrutura: na versão beta-2, havia um modelo de fenômenos de enfermagem e outro de ações, cada um deles com oito eixos e, na atual, os modelos foram unificados, formando apenas um modelo com sete eixos ${ }^{(7)}$.

Este estudo analisa o primeiro eixo do novo modelo, o foco da prática, Que contém 816 termos.

\section{CIPESC ${ }^{\circledR}$}

Durante o desenvolvimento da CIPE ${ }^{\circledR}$, o CIE identificou que a mesma não contemplava termos relacionados à atenção primária $\mathrm{e}$ à prática de enfermagem em serviços comunitários de saúde. Neste sentido, o conselho propôs aos países da América Latina a organização e identificação de termos característicos de cada realidade e região. A contribuição brasileira a esta proposta foi o projeto CIPESC, elaborado e desenvolvido pela ABEn (Associação Brasileira de Enfermagem), sob orientação do CIE e apoio financeiro da Fundação Kellogg ${ }^{(17)}$.

O principal objetivo do CIPESC foi descrever as diferenças culturais, revelando a dimensão, a diversidade e a amplitude das práticas de enfermagem no contexto do Sistema Único de Saúde SUS ${ }^{(18)}$. Para viabilizar a descrição do processo de trabalho da enfermagem, o Brasil foi dividido em cenários. O material produzido foi analisado em duas etapas e, como resultado obtiveram-se um inventário vocabular e, posteriormente, uma classificação denominada CIPESC $^{\circledR}$.

A CIPESC ${ }^{\circledR}$ teve como base a CIPE $^{\circledR}$ versão beta e, na sua

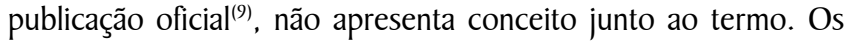
termos específicos desta classificação estão parcialmente conceituados na publicação de Garcia, Nóbrega e Sousa ${ }^{(19)}$.

\section{METODOLOGIA}

Esta investigação configura-se em um estudo descritivo documental de abordagem Quantitativa.

As bases empíricas utilizadas foram: a versão beta-2 e versão 1.0 da CIPE $^{\circledR}$ (edição Portuguesa e Brasileira), a CIPESC $^{\circledR}$, um trabalho de equivalência semântica ${ }^{(19)}$, um dicionário de termos de enfermagem $^{(20)}$ e dicionários da língua portuguesa ${ }^{(21,22)}$.

Primeiramente, o mapeamento consistiu em localizar os termos da CIPESC $^{\circledR}$ e da CIPE $^{\circledR}$ beta-2 na CIPE ${ }^{\circledR}$ 1.0. Concluída a localização dos termos, os mesmos foram classificados como: termo novo; termo com conceito idêntico; termo com conceito ampliado; termo com conceito diminuído; termo com conceito diferente; termo modificado; termo com conceito na beta 2 e sem conceito na versão I.0. Os resultados são apresentados por freQüência absoluta e percentual simples.

\section{RESULTADOS E DISCUSSÃO}

O Gráfico I representa o mapeamento dos termos da $\mathrm{CIPE}^{\circledR}$ I.0 em relação aos da beta- 2 .

O eixo, foco da prática na CIPE ${ }^{\circledR} 1.0$, apresenta 816 termos; destes, $41 \%$ são termos novos, $33 \%$ são termos idênticos entre as versões. Os termos restantes estão divididos em: $4 \%$ dos termos com conceito ampliado; 3\% dos termos com conceito diminuído;

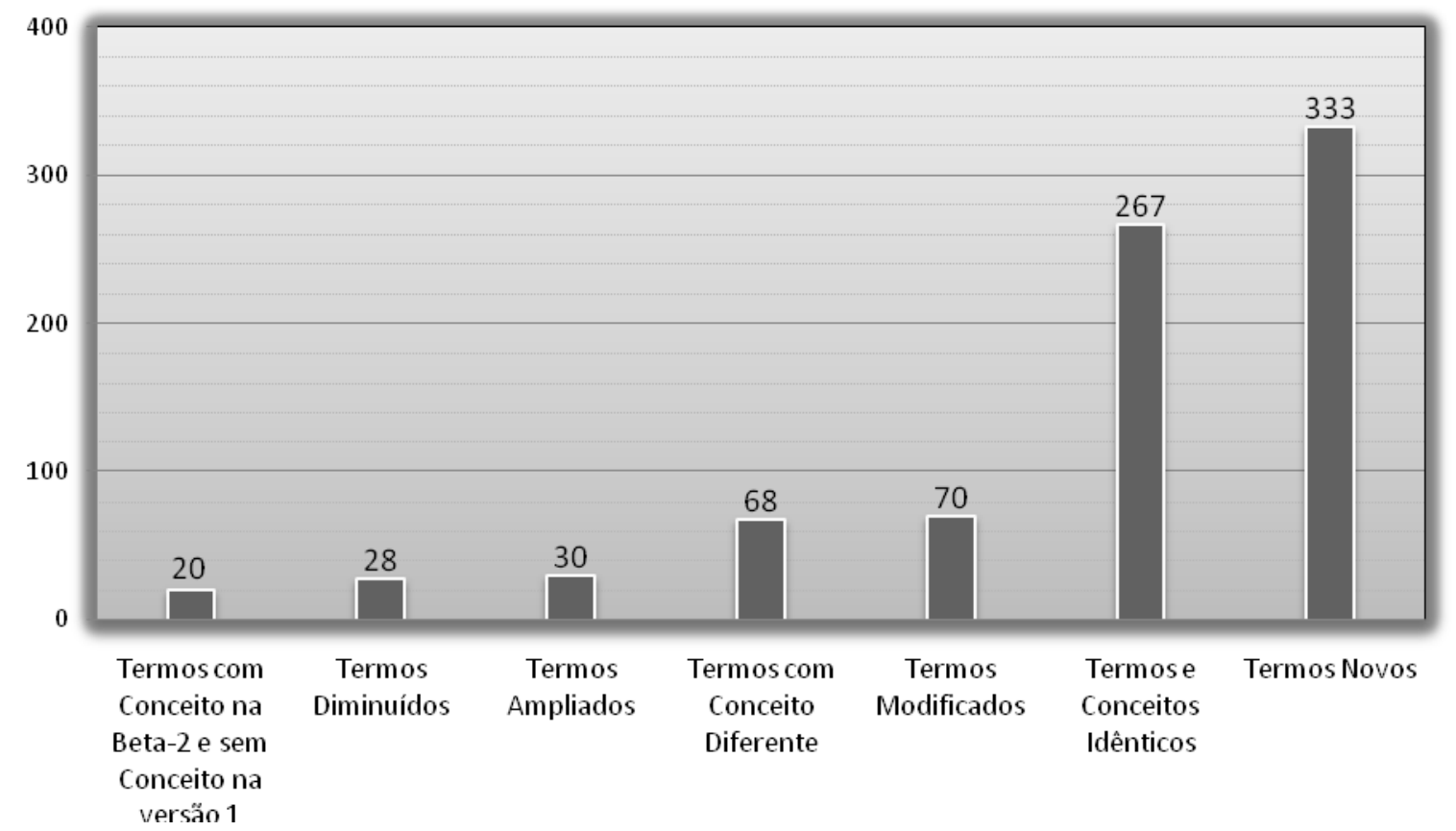

Gráfico 1. Freeüência absoluta da correlação entre os termos da $\mathrm{CIPE}^{\circledast} 1.0$ com beta-2. $(\mathrm{N}=816)$. 
$8 \%$ dos termos com conceito diferente, $9 \%$ dos termos modificados e $2 \%$ dos termos com conceito na beta-2 e sem conceito na versão 1.0 .

A organização dos resultados está exemplificada nos Quadros 1, 2, 3 e 4. As alterações dos conceitos estão destacadas em negrito e sublinhadas. O encuadramento dos termos foi realizado, primariamente, pelas modificações em negrito. As palavras sublinhadas podem apresentar mais de uma categorização e, por constituírem ambigüidades, serão submetidas à posterior análise e validação por especialistas.

O Quadro I representa os termos com conceito ampliado, considerados àQueles com acréscimo de uma ou mais palavras ao conceito dos termos.

Nota-se que QualQuer mudança de palavra pode influenciar no sentido do termo, como visualizado no termo 2 do Quadro 1. Inicialmente, abuso conjugal foi classificado como conceito igual; no entanto, o sentido da palavra conjugal é maior do que da palavra esposo(a) ${ }^{(21,22)}$, portanto ampliado.

O Quadro 2 apresenta os termos classificados como diminuídos, de cuja definição foram retiradas uma ou mais palavras.

No Quadro 2, a exclusão de algumas palavras não influencia diretamente no significado do termo. Porém, Quando se faz uma análise superficial dos termos sublinhados, é possível verificar que a troca de uma palavra por outra, teoricamente parecida, sugere cautela. À exemplo: nas expressões "animal manso" e "animal domesticado" (Quadro 2, termo I) os adjetivos têm similaridade; no entanto, as palavras "violadas" e "atacadas" (Quadro 2, termo
3) não possuem o mesmo significado. Desta forma, o Que determinou a inclusão do termo em uma ou outra classe foi a preservação do sentido do mesmo.

O Quadro 3 apresenta termos cujo sentido foi modificado.

Os termos 1 e 2 do Quadro 3 possuem alterações em palavras, as Quais modificaram o conceito do termo. Os termos 3 e 4 do mesmo Quadro apresentam conceitos divergentes.

Outro fato a ser considerado nesta organização diz respeito à hierarQuia do termo: o termo "energia" (Quadro 3, termo 4), na versão beta-2, pertence ao "Ser humano" e, na versão 1.0, ao "Ambiente", o Que pode revelar inconsistências nas combinações entre os mesmos pelo fato de serem termos idênticos, no entanto relacionados a taxonomias diferentes, o Que muitas vezes passa despercebido para os profissionais não especialistas em classificações.

O Quadro 4 exemplifica termos cujo conceito possui palavras diferentes, mas com preservação de significado.

Para organização dos termos exemplificados no Quadro 4, foi considerada a relação direta de significado das palavras diferentes, cujos conceitos nos dicionários de língua pátria apresentaram equivalência.

Os resultados do mapeamento dos termos das classificações CIPE $^{\circledR} 1.0$ com CIPESC ${ }^{\circledR}$ são apresentados no Gráfico 2.

Identificou se Que 79\% dos termos não são encontrados na CIPESC $^{\circledR}$ e $8 \%$ são termos idênticos. Os $13 \%$ restantes foram categorizados como: $1 \%$ termos com conceito ampliado; $2 \%$ termos com conceito diminuído; $4 \%$ termos com conceito diferente; $3 \%$

\begin{tabular}{|l|l|l|}
\hline Termo & CIPE $^{\circledR}$ versão betz2 & CIPE $^{\circledR}$ versão I \\
\hline I. Infanticídio & $\begin{array}{l}\text { (..) práticas tradicionais de matar o bebê logo } \\
\text { após o nascimento, em algumas sociedades (...) }\end{array}$ & $\begin{array}{l}\text { (...) práticas tradicionais de matar o bebê logo após o } \\
\text { nascimento, presentes em algumas sociedades (...) }\end{array}$ \\
\hline 2. Abuso Conjugal & $\begin{array}{l}\text { Ato de violar, atacar e maltratar o(a) esposo(a), } \\
\text { associado a comportamentos (...) }\end{array}$ & $\begin{array}{l}\text { Ato de violência, ataque e maltrato conjugal, associado } \\
\text { com comportamento (...) }\end{array}$ \\
\hline 3. Exercitar & $\begin{array}{l}\text { Realizar atividades físicas e programas de } \\
\text { exercícios corporais com o objetivo de manter a } \\
\text { forma, mobilidade e saúde. }\end{array}$ & $\begin{array}{l}\text { Desempenhar atividades físicas e programas de } \\
\text { exercícios corporais com o objetivo de manter a boa } \\
\text { forma física, a mobilidade e a saúde. }\end{array}$ \\
\hline 4. Uso de álcool & $\begin{array}{l}\text { Uso regular de álcool como estimulante, } \\
\text { normalmente bebendo vinho, cerveja ou licor. }\end{array}$ & $\begin{array}{l}\text { Uso regular de álcool como estimulante, } \\
\text { freeüentemente em bebidas como vinho, cerveja ou } \\
\text { licor. }\end{array}$ \\
\hline
\end{tabular}

\section{Quadro 1. Termos com conceito ampliado.}

\begin{tabular}{|l|l|l|}
\hline \multicolumn{1}{|c|}{ Temo } & \multicolumn{1}{|c|}{ CIPE $^{\circledR}$ versão betz2 } & \multicolumn{1}{c|}{ CIPE $^{\circledR}$ versão I } \\
\hline I. Animal doméstico & $\begin{array}{l}\text { Animal manso pertencente a um dono, } \\
\text { influenciando a vida e o desenvolvimento de } \\
\text { seres humanos. }\end{array}$ & Animal domesticado, pertencente a um proprietário. \\
\hline 2. Hipertensão & $\begin{array}{l}\text { Bombeamento do sangue para os vasos } \\
\text { sanguíneos com pressão (...) }\end{array}$ & $\begin{array}{l}\text { Bombeamento do sangue através dos vasos com } \\
\text { pressão (...) }\end{array}$ \\
\hline 3. Assédio Sexual & $\begin{array}{l}\text { Ações sexualmente motivadas de violência } \\
\text { verbal ou física de uma ou mais pessoas que } \\
\text { exercem poder e reduzem o direito das vítimas } \\
\text { de igualdade de oportunidade privacidade e } \\
\text { liberdade de não serem violadas. }\end{array}$ & $\begin{array}{l}\text { Aços sexualmente motivadas de violações físicas ou } \\
\text { verbais de uma ou mais pessoas Que atuam exercendo } \\
\text { poder e limitando os direitos de igualdade da vítima, de } \\
\text { privacidade e de liberdade de não ser atacada. }\end{array}$ \\
\hline 4. Vínculo & $\begin{array}{l}\text { Ligação entre uma criança e os pais; formação } \\
\text { de laços afetivos desde o nascimento, } \\
\text { consumindo-se gradualmente durante a infância. }\end{array}$ & $\begin{array}{l}\text { Ligação entre uma criança e os pais; formação de laços } \\
\text { afetivos. }\end{array}$ \\
\hline
\end{tabular}

Quadro 2. Termos com conceito diminuído. 


\begin{tabular}{|l|l|l|}
\hline \multicolumn{1}{|c|}{ Termo } & \multicolumn{1}{|c|}{ CIPE $^{\circledR}$ versão beta2 } & \multicolumn{1}{c|}{ CIPE $^{\circledR}$ versão I } \\
\hline I. Abuso Sexual & $\begin{array}{l}\text { Maltrato sexual ou ataque, participação } \\
\text { voluntária ou forçada em atos sexuais (...) }\end{array}$ & $\begin{array}{l}\text { Ataque ou maltrato sexual, participaça forçada em } \\
\text { carícias ou atos sexuais (...) }\end{array}$ \\
\hline 2. Violência & $\begin{array}{l}\text { (...) ações de violência, ataque ou prejuízo, (...) } \\
\text { estado de poder, luta ou conflito. }\end{array}$ & $\begin{array}{l}\text { (...) ações de violência, ataque ou prejuízo, (...) conflito } \\
\text { pelo poder, luta ou conflito. }\end{array}$ \\
\hline 3. Entidade Ambiental & $\begin{array}{l}\text { Condição ou influência sob as Quais os seres } \\
\text { humanos vivem ou se desenvolvem }\end{array}$ & $\begin{array}{l}\text { O complexo de entidades físicas, Químicas, bióticas, } \\
\text { sociais e culturais que influenciam a vida do individuo } \\
\text { ou comunidade. }\end{array}$ \\
\hline 4. Energia & $\begin{array}{l}\text { Disposição para demonstrar capacidade e } \\
\text { vontade para assumir ações, trabalhar e realizar } \\
\text { atividades vigorosas. }\end{array}$ & Fonte de força utilizável. \\
\hline
\end{tabular}

Quadro 3. Termos com conceito diferente.

\begin{tabular}{|l|l|l|}
\hline \multicolumn{1}{|c|}{ Termo } & \multicolumn{1}{|c|}{ CIPE $^{\circledR}$ versão betz2 } & \multicolumn{1}{c|}{ CIPE $^{\circledR}$ versão I } \\
\hline I. Animal Selvagem & $\begin{array}{l}\text { Animal indomável, não-domesticado e animal } \\
\text { não controlado (...) }\end{array}$ & Animal feroz, não domesticado e não controlado (...) \\
\hline 2. Leis de Trabalho Infantil & $\begin{array}{l}\text { Existência de normas Que estabelecem a idade } \\
\text { mínima (...) }\end{array}$ & Existência de padrões Que ditam a idade mínima (...) \\
\hline 3. Uso de Substâncias & $\begin{array}{l}\text { Uso regular de substâncias (...) e pode causar } \\
\text { dependência. }\end{array}$ & $\begin{array}{l}\text { Uso regular de substâncias (...) Que podem ser } \\
\text { prejudiciais à saúde e causar vício. }\end{array}$ \\
\hline 4.Choque & Falência circulatória (...) & Insuficiência circulatória (...) \\
\hline
\end{tabular}

Quadro 4. Termos com palavras diferentes e conceito equivalente.

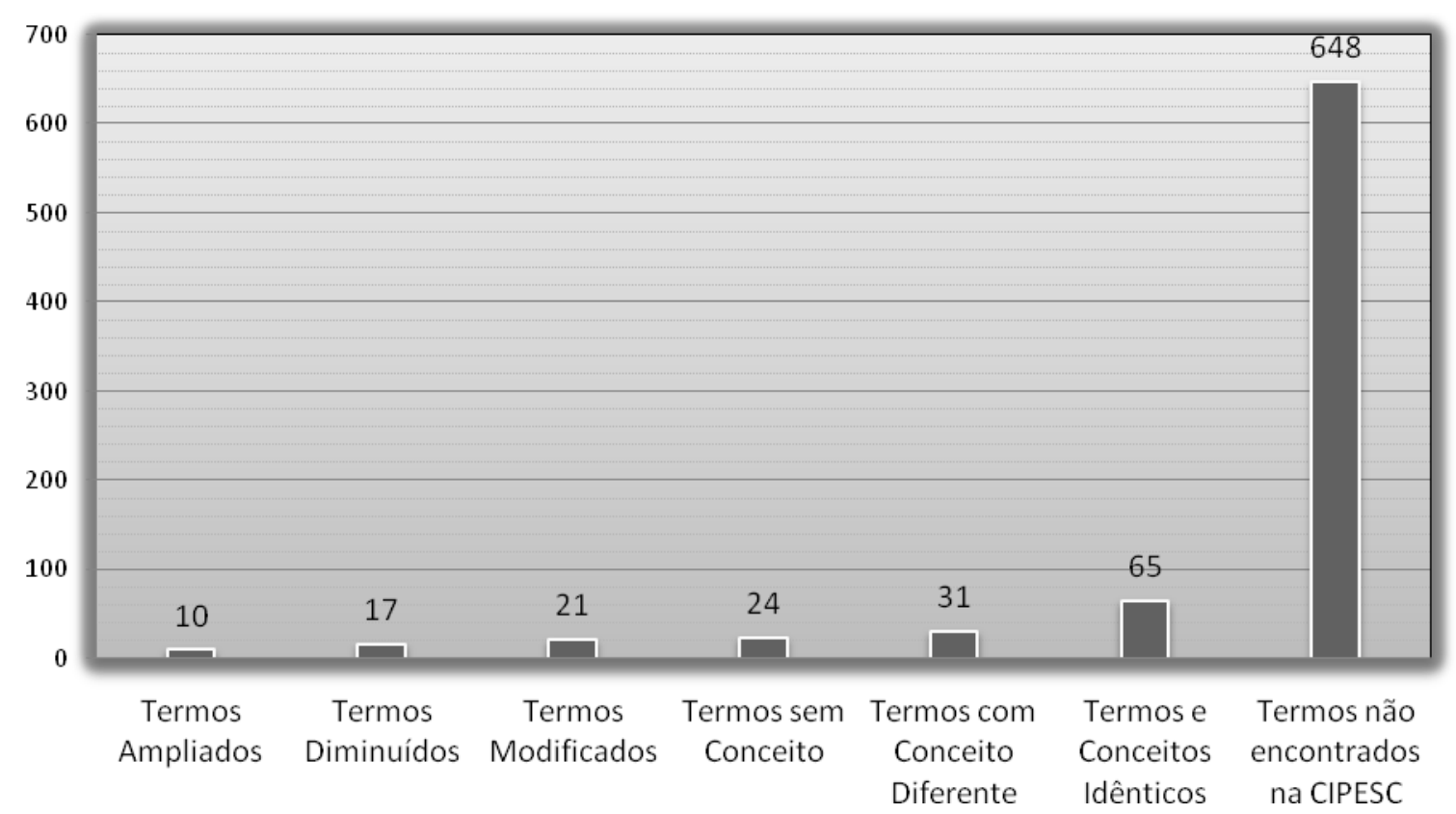

Gráfico 2. Freeüência absoluta da correlação entre os termos da $\operatorname{CIPE}^{\circledR} 1 \operatorname{com} \operatorname{CIPESC}^{\circledR} .(\mathrm{N}=816)$.

termos modificados com conceito equivalente; e 3\% termos sem conceito.

Cabe ressaltar Que, neste mapeamento, inicialmente foram identificados 11 termos na CIPE I.0 Que originalmente se situavam dentre os 131 termos específicos da $\operatorname{CIPESC}^{\circledR}$. Estes serão submetidos à posterior validação por especialistas.

\section{CONSIDERAÇÕES FINAIS}

A busca por uma classificação universal na enfermagem é processo em construção. A fim de alcançar este objetivo, revisões e inovações são incorporadas à CIPE ${ }^{\circledR}$ para reduzir a ambigüidade e redundância. Da mesma forma, para CIPESC $^{\circledR}$ acompanhar a 
evolução da CIPE $^{\circledR}$, buscou-se neste trabalho construir um mapeamento entre os termos do foco da prática da CIPE $^{\circledR}$ beta 2 , versão 1.0 e da CIPESC $^{\circledR}$, identificando semelhanças e diferenças entre os mesmos.

O mapeamento de termos foi produto de trabalho minucioso. Dúvidas foram levantadas, pesQuisas foram necessárias, bem como revisões constantes de todo o processo de localização e classificação dos mesmos.

Houve dificuldades operacionais derivadas da CIPE ${ }^{\circledR}$ versão 1.0 brasileira, Que apresenta falhas no processo de tradução e editoração, o Que pode levar o leitor e pesquisador a uma interpretação errônea do termo ou do próprio conceito.
Em relação à CIPESC ${ }^{\circledR}$, o agravante foi o fato da mesma não possuir a totalidade de seus termos conceituados, dificultando a correspondência com outros termos.

Este trabalho possibilitou identificar correlações entre as classificações CIPE ${ }^{\circledR}$ e CIPESC ${ }^{\circledR}$. Este resultado, em continuidade à pesquisa em Que se insere, desencadeará um processo de validação para os termos não equivalentes. Posteriormente, esta equivalência será imprescindível para o aprimoramento da ferramenta CIPESC ${ }^{\circledR}$ com o desenvolvimento de uma ontologia, a Qual, futuramente, será integrada a um sistema de informação, viabilizando não apenas o crescimento da classificação, mas também o apoio computacional ao diagnóstico de enfermagem.

\section{REFERÊNCIAS}

1. Antunes MIM, Chianca TCM. As classificações de enfermagem na saúde coletiva- o projeto CIPESC ${ }^{\circledR}$. Rev Bras de Enferm 2002; 55 (6): 644-5I.

2. Horta WA, Castellanos BP. Processo de enfermagem. São Paulo (SP): 1979

3. Ferreira NMLA. Sistematização da assistência de enfermagemimportância para a profissão e responsabilidade no preparo do enfermeiro. Acta Paul Enferm 1990; 3 (3): 79-84.

4. Nóbrega MML, Gutiérrez MGR. Equivalência semântica da Classificação de Fenômenos de Enfermagem da CIPE ${ }^{\circledR}$ Versão Alfa. João Pessoa: Idéia; 2000.

5. Guimarães HCQCP, Barros ALBL, Gutierrez MGR. Identificação das características definidoras do diagnóstico de enfermagem excesso de volume de líquidos. Rev Latino-Am Enfermagem 2000; 8 (2): 68-73.

6. Nobrega MML, Garcia TR. Terminologias em enfermagem: desenvolvimento e perspectivas de incorporação na prática profissional. In: Albueuerque LM, Cubas MR. Cipescando em Curitiba: construção e implementação da nomenclatura de diagnósticos e intervenções de enfermagem na rede básica de saúde. Curitiba: ABEn; 2005.

7. International Council of Nurses. Geneve: 2008. Disponível em: http://www.icn.ch/icnp_vl book_ch4.htm

8. Camiá GEK, Barbieri M, Marin HF. Fenômenos de enfermagem identificados em consultas de planejamento familiar segundo a ICNP ${ }^{\circledR}$ - Versão Beta 2. Rev Latino-Am Enfermagem 2006; 14 (5).

9. Nielsen GH, Mortensen R. Classificação Internacional das práticas de enfermagem do Conselho Internacional de Enfermeiras: versão alpha. Brasília: ABEn; 1997.

10. Conselho Internacional de Enfermagem. Classificação Internacional das Práticas de Enfermagem: versão beta-2. São Paulo: UNIFESP; 2003.

II. Conselho Internacional de Enfermagem. Classificação Internacional das Práticas de Enfermagem: versão I.0. São Paulo: Algol; 2007.
12. Gruber TR. What is an Ontology? 1992. Disponível em: http:/ /www-ksl.stanford.edu/kst/what-is-an-ontology.html

13. Garcia TR, Nóbrega MML, organizadores. Sistemas de Classificação em Enfermagem: um trabalho coletivo. João Pessoa: Idéia; 2000.

14. Theune VB, Basso X, Henriquez PC, Pedreros MC. La Clasificación Internacional para la Práctica de Enfermería como ejemplo de Investigación em Red. Ciencia y Enfermeria 2004; 10 (2): 9-I3.

15. Nóbrega MML, Gutiérrez MGR. Sistemas de Classificação na Enfermagem: avanços e perspectivas. In: Garcia TR, Nobrega MML, organizadores. Sistemas de Classificação em Enfermagem: um trabalho coletivo. João Pessoa (PB): Idéia; 2000. p.1-15.

16. Nobrega MML, Garcia TR. Perspectivas de incorporação da Classificação Internacional para a Prática de Enfermagem (CIPE ${ }^{\circledR}$ ) no Brasil. Rev Bras Enferm 2005; 58 (2): 227-30.

17. Coenen A, Marin HF, Park HA, Bakken S. Collaborative efforts for representig nursing concepts in computer-based systems: International perspectives. I Am Med Inform Assoc 200 I; 8 (3): 202-11.

18. Egry E, Mishima S, Antunes MJM. Classificação das práticas de enfermagem em saúde coletiva: a experiência brasileira. In: Chianca TCM, Antunes MJM. A Classificação Internacional das Práticas em Saúde Coletiva. CIPESC. Brasília: ABEn; 1999.

19. Garcia TR, Nóbrega MML, Sousa MCM. Validação das definições de termos identificados no projeto CIPESC para o eixo foco da prática em enfermagem da CIPE. Rev Bras Enferm 2002; 55 (1): 52- 63.

20. Costa F. Grande dicionário de enfermagem atual. Rio de Janeiro: Robe Editorial; 2000.

21. Ferreira $A B H$. Aurélio século XXI: o dicionário da língua portuguesa. Rio de Janeiro: Nova Fronteira, 1999.

22. Priberam. Dicionário da Língua Portuguesa On-line. Disponível em: URL: http://www.priberam.pt/dlpo/dlpo.aspx 